\title{
Absolute root separation
}

\author{
Yann Bugeaud, Andrej Dujella, Wenjie Fang, \\ Tomislav Pejković, and Bruno Salvy
}

December 2, 2019

\begin{abstract}
The absolute separation of a polynomial is the minimum nonzero difference between the absolute values of its roots. In the case of polynomials with integer coefficients, it can be bounded from below in terms of the degree and the height (the maximum absolute value of the coefficients) of the polynomial. We improve the known bounds for this problem and related ones. Then we report on extensive experiments in low degrees, suggesting that the current bounds are still very pessimistic.
\end{abstract}

\section{Separation and absolute separation}

The absolute separation of a polynomial $P \in \mathbb{C}[X]$ is the minimal nonzero distance between the absolute values of its complex roots:

$$
\operatorname{abs} \operatorname{sep}(P):=\min _{\substack{P(\alpha)=P(\beta)=0,|\alpha| \neq|\beta|}}|| \alpha|-| \beta|| .
$$

Having good lower bounds on this quantity for polynomials with integer coefficients is of interest in the asymptotic analysis of linear recurrent sequences.

To the best of our knowledge, the first published bound on this problem 7 ] was abs $\operatorname{sep}(P) \gg \mathrm{H}(P)^{-d\left(d^{2}+2 d-1\right) / 2}$ where, here and below, the constant implicit in the $\gg$ sign depends only on the degree $d$, while $\mathrm{H}(P)$, the height of the polynomial $P$, is the maximum of the absolute values of its coefficients. This exponent was later [4, 15] improved to $-d^{3} / 2+d^{2}+d / 2-2$ and even more recently [3] to $-d^{3} / 2+d^{2}+d / 2-1$. In this work, we improve this exponent and that of related problems. Still, we do not know how far the exponent we obtain is from being optimal. Thus an important part of this article is devoted to experiments in low degree, from where we can infer families of polynomials exhibiting a behaviour in $\mathrm{H}(P)^{-d-1}$ for $d \in\{3,4,5,6\}$.

In the much more classical case of the separation

$$
\operatorname{sep}(P):=\min _{\substack{P(\alpha)=P(\beta)=0, \alpha \neq \beta}}|\alpha-\beta|,
$$


the best available bound for a polynomial with integer coefficients goes back to Mahler [10]:

$$
\operatorname{sep}(P) \gg \mathrm{H}(P)^{-d+1} \text {. }
$$

Even in that case, the tightness of the exponent $-d+1$ is still unknown, with best known upper bounds $-(2 d-1) / 3$ for general $d$ and -2 for $d=3$ [1, 5, 14]. (A consequence noted by Mahler is that the right-hand side of (11) also gives a lower bound on the absolute value of the imaginary parts of nonreal roots of $P$.)

This work consists of two parts. In the first one, we improve the known exponents for the absolute separation and related problems with the following.

Theorem 1. Let $P \in \mathbb{Z}[X]$ be a polynomial of degree $d$ and let $\alpha$ and $\beta$ be two of its roots such that $|\alpha| \neq|\beta|$, then

1. if $\alpha$ and $\beta$ are real, then ||$\alpha|-| \beta|| \gg \mathrm{H}(P)^{-(d-1)}$;

2. if $\alpha$ is real and $\beta$ is not, then ||$\alpha|-| \beta|| \gg \mathrm{H}(P)^{-2(d-1)(d-2)}$;

3. if neither of them is real, then ||$\alpha|-| \beta|| \gg \mathrm{H}(P)^{-(d-1)(d-2)(d-3) / 2}$.

We proved a more precise version of the first bound in a previous work [2], where we showed that the exponent of $\mathrm{H}(P)$ is optimal in that case. More detailed but less precise bounds for the second case can be found in [4, Lemma 2.5], [11, Lemma 3.6] and [12, Lemma 53]. Note that the third case requires $d \geq 4$ to be meaningful, since a cubic polynomial with real coefficients cannot have two non-real roots with distinct absolute values.

The proof of Theorem 1 is based on constructing auxiliary polynomials with integer coefficients of controlled height whose roots contain the desired difference. This is a very versatile approach. We illustrate it to rederive Mahler's exponent $-d+1$ in Section 2.1. Next, we apply it to the three cases covered in Theorem 1. Similar bounds for the difference between the real or the imaginary parts of roots of integer polynomials are derived in Sections 2.4 and 2.5 .

In the second part of this work (Section 3), we describe experiments leading to lower bounds on the absolute separation for small degrees, which can be summarized as follows.

Theorem 2. For each $d \in\{3,4,5,6\}$, there exists a sequence $\left(P_{d, M}\right)$ of polynomials of degree $d$ in $\mathbb{Z}[X]$, such that as $M \rightarrow \infty$, the polynomial $P_{d, M}$ has two roots $\alpha_{M}, \beta_{M}$ with $\left|\alpha_{M}\right| \neq\left|\beta_{M}\right|$ and

$$
|| \alpha_{M}|-| \beta_{M}|| \ll \mathrm{H}\left(P_{d, M}\right)^{-d-1}, \quad M \rightarrow \infty .
$$

Thus apart from its first part, $d=3$ is the only case where we know Theorem 1 to be optimal. It is interesting to note that, in our examples, the growth of $H\left(P_{3, M}\right)$ is exponential in $M$, while that of $H\left(P_{d, M}\right)$, for $d \in\{4,5,6\}$, is linear in $M$. 


\section{General bounds from symmetric functions of roots}

Here and in the rest of this article, we consider a polynomial $P(X) \in \mathbb{Z}[X]$,

$$
P(X)=\sum_{i=0}^{d} a_{i} X^{i}=a_{d} \prod_{i=1}^{d}\left(X-\alpha_{i}\right),
$$

of degree $d\left(a_{d} \neq 0\right)$ with coefficients of absolute value bounded by $H$ and complex roots $\alpha_{1}, \ldots, \alpha_{d}$.

Our bounds on various types of separations that are asymptotic in the height of the polynomial are obtained from the following two classical results, of which we sketch the proofs for completeness.

Proposition 1 (Effective Version of the Fundamental Theorem of Symmetric Functions). [16, Thm. 6.21] Let $P(X)=\sum_{i=0}^{d} a_{i} X^{i}=a_{d} \prod_{i=1}^{d}\left(X-\alpha_{i}\right) \in$ $\mathbb{Z}\left[a_{0}, \ldots, a_{d}, X\right]$ and let $G \in \mathbb{Z}\left[X_{1}, \ldots, X_{d}\right]$ be a symmetric polynomial of degree at most $k$ in each $X_{i}$. Then $a_{d}^{k} G\left(\alpha_{1}, \ldots, \alpha_{d}\right)$ is a polynomial of total degree at most $k$ in $\mathbb{Z}\left[a_{0}, \ldots, a_{d}\right]$.

This is a standard result on symmetric functions [9, Ch. 1]. We now give an elementary self-contained proof.

Proof. Let $b_{i}=a_{d-i} / a_{d}$ for $1 \leq i \leq d$. These are algebraically independent symmetric functions of the $\alpha_{j}$ 's. We need to prove that $G\left(\alpha_{1}, \ldots, \alpha_{d}\right)$ is a polynomial of total degree at most $k$ in $\mathbb{Z}\left[b_{1}, \ldots, b_{d}\right]$. By linearity, it is sufficient to consider the case where $G$ is homogeneous of total degree $k$. Let $\Lambda_{k}$ be the linear space of such symmetric polynomials in $\alpha_{1}, \ldots, \alpha_{d}$.

Since $b_{i}$ is of total degree $i$ in the $\alpha_{j}$ 's, all products of the form

$$
e_{\mu}=b_{1}^{\mu_{1}} b_{2}^{\mu_{2}} \cdots b_{d}^{\mu_{d}}
$$

indexed by $\mu=\left(\mu_{i}\right)$ satisfying $\sum_{i=1}^{d} i \mu_{i}=k$ are linearly independent and in $\Lambda_{k}$. Such a product has total degree $\sum_{i=1}^{d} \mu_{i} \leq k$ in the $b_{i}$ 's. However, $\Lambda_{k}$ is also linearly generated by the monomials of the form

$$
m_{\lambda}=\sum_{\sigma \in S_{d}} \alpha_{\sigma(1)}^{\lambda_{1}} \cdots \alpha_{\sigma(d)}^{\lambda_{d}}
$$

indexed by $\lambda=\left(\lambda_{j}\right)$ with $\lambda_{1} \geq \lambda_{2} \geq \cdots \geq \lambda_{d}$ and $\sum_{j=1}^{d} \lambda_{j}=k$. The dimension is given by the number of distinct such $\left(\lambda_{j}\right)$, which is also the number of $\left(\mu_{i}\right)$, where each $\mu_{i}=\lambda_{i}-\lambda_{i+1}$. Therefore, the $e_{\mu}$ 's also linearly span $\Lambda_{k}$ and $G$ can be written as a linear combination of $e_{\mu}$ 's with rational coefficients.

To prove that the coefficients are integers, we order the monomials in $\alpha_{j}$ 's lexicographically. Since $b_{i}$ is the sum of $\prod_{j \in S} \alpha_{j}$ over all subsets $S$ of $\{1, \ldots, d\}$ with $i$ elements, the largest monomial in $e_{\mu}$ is given by $\alpha_{1}^{\mu_{1}^{\prime}} \cdots \alpha_{d}^{\mu_{d}^{\prime}}$, where 
$\mu_{j}^{\prime}=\sum_{i=j}^{d} \mu_{i}$. The map $\mu \mapsto \mu^{\prime}=\left(\mu_{j}^{\prime}\right)$ is a bijection, which implies that every $e_{\mu}$ has a distinct monic leading monomial and all other monomials have integral coefficients. Induction in the lexicographical order finally shows that the coefficients of $G$ in the basis $\left(e_{\mu}\right)_{\mu}$ are all integers.

Proposition 2 (Cauchy Bound). [13, Thm. 4.2 (ii)] Let $P(X)=\sum_{i=0}^{d} a_{i} X^{i} \in$ $\mathbb{Z}[X]$ with $\left|a_{i}\right| \leq H$ for all $i$ and let $\alpha \neq 0 \in \mathbb{C}$ be one of its roots. Then

$$
|\alpha| \geq \frac{1}{1+H}
$$

Proof. We may assume $|\alpha|<1$, otherwise the result is obvious, and $a_{0} \neq 0$. Then, we get

$$
1 \leq\left|a_{0}\right| \leq \sum_{i=1}^{d}\left|a_{i}\right| \cdot\left|\alpha^{i}\right| \leq H \frac{|\alpha|}{1-|\alpha|},
$$

giving at once the lower bound $|\alpha| \geq \frac{1}{1+H}$.

\subsection{Motivating example: Mahler's bound}

Mahler's bound is usually deduced from Hadamard's bound on Sylvester's matrix applied to the discriminant of the polynomial. When only the asymptotic exponent of $\mathrm{H}(P)$ in the estimate is needed, Propositions 1 and 2 are sufficient. Indeed, consider the polynomial

$$
M(X)=a_{d}^{2(d-1)} \prod_{i<j}\left(X-\left(\alpha_{i}-\alpha_{j}\right)^{2}\right) .
$$

It is symmetric in the $\alpha_{i}$ 's, with degree in each $\alpha_{i}$ that is twice the number of $j \neq i$, i.e., $2(d-1)$. Thus, by Proposition 1, the polynomial $M(X)$ has integer coefficients of height bounded by $c H^{2(d-1)}$ for some constant $c$ that depends only on $d$. By Proposition 2, we thus get that for any $(i, j)$ such that $\alpha_{i} \neq \alpha_{j}$,

$$
\left|\alpha_{i}-\alpha_{j}\right|^{2} \geq \frac{1}{1+c H^{2(d-1)}} .
$$

Choosing $(i, j)$ that minimizes $\left|\alpha_{i}-\alpha_{j}\right|$ and taking square roots thus gives

$$
\operatorname{sep}(P) \gg H^{-(d-1)},
$$

recovering the exponent in Mahler's bound.

This method could be refined to produce a bound rather than an asymptotic estimate, by a more precise analysis of the coefficients of the polynomial involved in Proposition 1

Note also that the polynomial obtained by considering the roots $\alpha_{i}+\alpha_{j}$ in place of $\alpha_{i}-\alpha_{j}$ in $M$ satisfies the same bounds. It follows that the sum of non-opposite roots of $P$ is also lower bounded by $H^{-(d-1)}$, giving part 1 of Theorem 1 . 


\subsection{Absolute real-complex gap}

Part 2 of the theorem is obtained by considering the polynomial

$$
R(X)=a_{d}^{2(d-1)(d-2)} \prod_{i<j, k \notin\{i, j\}}\left(X-\left(\alpha_{k}^{2}-\alpha_{i} \alpha_{j}\right)\right) .
$$

This polynomial is symmetric in the $\alpha_{i}$ 's, of degree $2(d-1)(d-2)$ in each of them. Thus by Propositions 1 and 2 , its smallest nonzero root has magnitude at least $H^{-2(d-1)(d-2)}$. In particular, if $\alpha$ is a real root of $P$ and $\beta$ a nonreal root such that $|\alpha| \neq|\beta|$, taking $\alpha_{k}=\alpha, \alpha_{i}=\beta$ and $\alpha_{j}=\bar{\beta}$ gives

$$
\left.\left|\alpha^{2}-\right| \beta\right|^{2} \mid \gg H^{-2(d-1)(d-2)} .
$$

If $|\alpha|+|\beta| \leq 2$, then dividing both terms gives a similar inequality for ||$\alpha|-| \beta||$.

Otherwise, it is only in the case when ||$\alpha|-| \beta|| \leq 1$ that we need a lower bound. Supposing first that $|\beta| \geq|\alpha|$, then we have $|\alpha|+1 \geq|\beta|$ and thus $|\alpha|+|\alpha|+1 \geq|\alpha|+|\beta|>2$ and so $|\alpha|>1 / 2$ while $2|\beta| \geq|\alpha|+|\beta|>2$ gives $|\beta|>1$. Thus, $1 /|\alpha \beta|<2$ and $1 /|\alpha|+1 /|\beta|<3$. The same bounds are obtained when supposing $|\alpha| \geq|\beta|$. Now, since $\alpha^{-1}$ and $\beta^{-1}$ are roots of the reciprocal polynomial $X^{d} P(1 / X)$ that has the same height as $P$, we also have

$$
\left.\left|\alpha^{-2}-\right| \beta\right|^{-2} \mid=\frac{|| \alpha|-| \beta||}{|\alpha||\beta|}\left(\frac{1}{|\alpha|}+\frac{1}{|\beta|}\right) \gg H^{-2(d-1)(d-2)},
$$

whence the conclusion by dividing out by the factor smaller than 6 .

\subsection{Absolute complex-complex gap}

We now analyse the polynomial

$$
S(X)=a_{d}^{(d-1)(d-2)(d-3)} \prod_{\substack{i<j, k<\ell,\{i, j\} \cap\{k, \ell\}=\emptyset}}\left(X^{1 / 2}-\left(\alpha_{i} \alpha_{j}-\alpha_{k} \alpha_{\ell}\right)\right) .
$$

Exchanging the pairs $(i, j)$ and $(k, \ell)$ shows that this is indeed a polynomial in $X$. It is invariant under permutations of the $\alpha_{i}$ 's. Its degree in $X$ is $\sim d^{4} / 4$, but its degree in each of the $\alpha_{i}$ 's is only $(d-1)(d-2)(d-3)$, corresponding to all the possible choices of the other $\alpha_{j}$ 's. If $\alpha$ and $\beta$ are two non-real roots of $P$ with $|\alpha| \neq|\beta|$, then taking $\alpha_{i}=\alpha, \alpha_{j}=\bar{\alpha}, \alpha_{k}=\beta, \alpha_{\ell}=\bar{\beta}$ and using Propositions 1 and 2 again gives

$$
\left(|\alpha|^{2}-|\beta|^{2}\right)^{2} \gg H^{-(d-1)(d-2)(d-3)} .
$$

Taking square roots divides the exponent by 2 , and then with the help of the reciprocal polynomial $X^{d} P\left(X^{-1}\right)$, the same argument as in the case of absolute real-complex gap leads to

$$
\operatorname{abs} \operatorname{sep}(P) \gg H^{-(d-1)(d-2)(d-3) / 2},
$$

which concludes the proof of Theorem 1, 


\subsection{Gap on the real parts of roots}

The same approach gives bounds on gaps between real parts of roots. There are again three cases: real-real, real-complex, complex-complex. The real-real case is simply the corresponding case in root separation, which is already known. For the real-complex case, we consider the following polynomial

$$
T_{1}(X)=a_{d}^{\frac{3}{2}(d-1)(d-2)} \prod_{i<j, k \notin\{i, j\}}\left(X-\left(\alpha_{i}+\alpha_{j}-2 \alpha_{k}\right)\right),
$$

while the complex-complex case relies on

$$
T_{2}(X)=a_{d}^{(d-1)(d-2)(d-3)} \prod_{\substack{i<j, k<\ell,\{i, j\} \cap\{k, \ell\}=\emptyset}}\left(X^{1 / 2}-\left(\alpha_{i}+\alpha_{j}-\alpha_{k}-\alpha_{\ell}\right)\right) .
$$

The analysis is as before. Both $T_{1}$ and $T_{2}$ can be seen to be polynomials in $X$. They are symmetric polynomials in the $\alpha_{i}$ 's. Their degrees in the $\alpha_{i}$ 's is the one used in the exponent of $a_{d}$. The smallest nonzero root of $T_{1}$ divided by 2 is a lower bound of the real-complex real-part gap, while $T_{2}$ gives the complexcomplex case. Propositions 1 and 2 then give the following.

Theorem 3. Let $P \in \mathbb{Z}[X]$ be a polynomial of degree $d$ and let $\alpha$ and $\beta$ be two of its roots such that $\Re \alpha \neq \Re \beta$, then

$$
|\Re \alpha-\Re \beta| \gg \begin{cases}\mathrm{H}(P)^{-(d-1)}, & \text { if } \Im \alpha=\Im \beta=0, \\ \mathrm{H}(P)^{-3(d-1)(d-2) / 2}, & \text { if } \Im \alpha=0, \\ \mathrm{H}(P)^{-(d-1)(d-2)(d-3) / 2}, & \text { otherwise. }\end{cases}
$$

\subsection{Gap on the imaginary parts of roots}

The situation for imaginary parts is similar. First, if one of the roots is real and the other is not, then as already mentioned, Mahler's bound applies to the imaginary part. If both roots are nonreal but not conjugates, then the same polynomial $T_{2}$ as in the case of the real parts can be used, with $\left(\alpha_{i}, \alpha_{k}\right)$ and $\left(\alpha_{j}, \alpha_{\ell}\right)$ taking the roles of conjugate roots. In the case when one of the roots is purely imaginary, then the following generalization of $T_{1}$ can be used

$$
T_{3}(X)=a_{d}^{3(d-1)(d-2)} \prod_{i<j, k \notin\{i, j\}}\left(X-\left(\alpha_{i}-\alpha_{j}-2 \alpha_{k}\right)^{2}\right) .
$$

This discussion leads to the following.

Theorem 4. Let $P \in \mathbb{Z}[X]$ be a polynomial of degree $d$ and let $\alpha$ and $\beta$ be two of its roots such that $\Im \alpha \neq \Im \beta$, then

$$
|\Im \alpha-\Im \beta| \gg \begin{cases}\mathrm{H}(P)^{-(d-1)}, & \text { if } \Im \alpha=0, \\ \mathrm{H}(P)^{-3(d-1)(d-2) / 2}, & \text { if } \Re \alpha=0, \\ \mathrm{H}(P)^{-(d-1)(d-2)(d-3) / 2}, & \text { otherwise. }\end{cases}
$$




\section{Experiments and bounds in low degree}

As already mentioned, even in the case of Mahler's bound, the tightness of the exponent is unknown. The situation is similar for the bounds obtained in the previous section.

We now turn to experiments that lead to lower bounds on the asymptotic separation. In order to obtain such an asymptotic result, we search for families of polynomials exhibiting a small absolute separation. In particular, we would like to approach a tight estimate in the case of low degrees, as a first step for a better understanding of the actual growth of these bounds with the degree. We use two complementary types of experiments.

Exhaustive search First, we perform an exhaustive search for polynomials of small absolute separation given a degree and a bound on the height. More specifically, given a degree, we search through all polynomials with integer coefficients within a given height, in case a pattern can be discerned in the polynomials with small absolute separation. In some cases indeed, the roots of these polynomials seem to concentrate in certain locations, letting us refine the experiment and search more closely. This is successful in degree 3, where we find a family of polynomials letting us prove the tightness of the exponent -4 in that case (Proposition 3 below). However, even if some time is saved by taking into account various symmetries, the number of polynomials to be tested is too large for this approach to be used for large height and even more so for large degree.

Perturbations While these exhaustive searches are purely numerical, our second type of experiments relies heavily on symbolic computation. In degree $d$, we consider polynomials of the form

$$
P_{d}(X, \epsilon)=R_{d}(X)+\epsilon Q_{d}(X) .
$$

Here, for a given $r \in \mathbb{N}^{+}, R_{d}(X)$ is a polynomial with several roots of absolute value $r$, having factors of the type $X \pm r$ and $X^{2}+a X+r^{2}$, with $a$ an integer such that $|a|<2 r$. The polynomial $Q_{d}(X)$ is subject to $\max \left(\operatorname{deg}\left(R_{d}\right), \operatorname{deg}\left(Q_{d}\right)\right)=d$ so that $P_{d}(X, \epsilon)$ has degree $d$ in $X$. For $X_{1}(\epsilon)$ and $X_{2}(\epsilon)$ two roots of $P_{d}(X, \epsilon)$ such that $X_{1}(0)$ and $X_{2}(0)$ are roots of selected factors of $R_{d}$, we compute a series expansion of these roots of $P_{d}$ in powers of $\epsilon$, from which we deduce

$$
\left|X_{i}(\epsilon)\right|^{2}=\sum_{k \geq 0} c_{i, k} \epsilon^{k} .
$$

This computation is purely symbolic, meaning that the $c_{i, k}$ 's are computed as explicit polynomials in the coefficients of $R_{d}$ and $Q_{d}$. We then look for integer solutions of the polynomial system $\left\{c_{1, k}=c_{2, k}, 1 \leq k \leq h-1\right\}$ that do not satisfy $c_{1, h}=c_{2, h}$. Such solutions give a polynomial $P_{d}(X, \epsilon)$ such that $\left|X_{2}(\epsilon)\right|^{2}-\left|X_{1}(\epsilon)\right|^{2}$ is not zero but is $O\left(\epsilon^{h}\right)$, so that the polynomial $P_{d}^{*}(X)=$ $M P_{d}\left(X, M^{-1}\right)$ with integer $M$ has integer coefficients and absolute separation $O\left(\mathrm{H}\left(P_{d}^{*}\right)^{-h}\right)$. 


\begin{tabular}{ccrc}
\hline $\begin{array}{c}\text { degree, } \\
\text { max height }\end{array}$ & $\begin{array}{c}\text { separation } \\
\text { type }\end{array}$ & $P(X)$ & separation \\
\hline 3,10 & $|\alpha-\beta|$ & $5 X^{3}+8 X^{2}-9 X+2$ & $1.421 \mathrm{e}-2$ \\
3,20 & $|\alpha-\beta|$ & $14 X^{3}+17 X^{2}-13 X+2$ & $4.938 \mathrm{e}-3$ \\
4,10 & $|\alpha-\beta|$ & $3 X^{4}-9 X^{3}-10 X^{2}+7 X-1$ & $4.187 \mathrm{e}-3$ \\
4,20 & $|\alpha-\beta|$ & $9 X^{4}-13 X^{3}-14 X^{2}+17 X-4$ & $5.974 \mathrm{e}-4$ \\
5,10 & $|\alpha-\beta|$ & $9 X^{5}+X^{4}-4 X^{3}-9 X^{2}-3 X+7$ & $4.656 \mathrm{e}-4$ \\
\hline 3,10 & ||$\alpha|-| \beta \mid$ & $10 X^{3}-3 X^{2}-2 X+3$ & $5.394 \mathrm{e}-4$ \\
3,20 & ||$\alpha|-| \beta \|$ & $17 X^{3}-9 X^{2}-7 X+8$ & $1.233 \mathrm{e}-5$ \\
4,10 & ||$\alpha|-| \beta \|$ & $X^{4}-6 X^{3}-7 X^{2}+5 X+6$ & $2.276 \mathrm{e}-6$ \\
4,20 & ||$\alpha|-| \beta \mid$ & $5 X^{4}-17 X^{3}-20 X^{2}+11 X+12$ & $1.034 \mathrm{e}-7$ \\
5,10 & ||$\alpha|-| \beta \|$ & $9 X^{5}-5 X^{4}-4 X^{3}-2 X^{2}-2 X-9$ & $1.459 \mathrm{e}-7$ \\
\hline 3,10 & $|\Re \alpha-\Re \beta|$ & $7 X^{3}+5 X^{2}+5 X+1$ & $5.952 \mathrm{e}-4$ \\
3,20 & $|\Re \alpha-\Re \beta|$ & $19 X^{3}+8 X^{2}+15 X+2$ & $2.218 \mathrm{e}-5$ \\
4,10 & $|\Re \alpha-\Re \beta|$ & $9 X^{4}+5 X^{3}-X^{2}+5 X-1$ & $1.472 \mathrm{e}-6$ \\
4,20 & $|\Re \alpha-\Re \beta|$ & $13 X^{4}+3 X^{3}+5 X^{2}+19 X-7$ & $1.669 \mathrm{e}-7$ \\
5,10 & $|\Re \alpha-\Re \beta|$ & $7 X^{5}-6 X^{4}-6 X^{3}-5 X^{2}+X+1$ & $2.511 \mathrm{e}-7$ \\
\hline 3,10 & $|\Im \alpha-\Im \beta|$ & $10 X^{3}+6 X^{2}-6 X+1$ & $2.403 \mathrm{e}-2$ \\
3,20 & $|\Im \alpha-\Im \beta|$ & $19 X^{3}+9 X^{2}-19 X+5$ & $5.082 \mathrm{e}-3$ \\
4,10 & $|\Im \alpha-\Im \beta|$ & $10 X^{4}+X^{3}+10$ & $6.250 \mathrm{e}-5$ \\
4,20 & $|\Im \alpha-\Im \beta|$ & $20 X^{4}+X^{3}+20$ & $7.813 \mathrm{e}-6$ \\
5,10 & $|\Im \alpha-\Im \beta|$ & $5 X^{5}-8 X^{4}+6 X^{3}+5 X^{2}-5 X+8$ & $1.061 \mathrm{e}-7$ \\
\hline
\end{tabular}

Table 1: Record polynomials for small degree and height

Results The rest of this section reports on these experiments. The results of an exhaustive search are first presented in 33.1 . Next, we discuss a family of polynomials of degree 3 proving the tightness of the exponent -4 by an argument we have been unable to generalize. In degrees 4,5 and 6 , the perturbative approach lets us find exponents $-5,-6,-7$.

\subsection{Exhaustive search for small degree and height}

The results of an exhaustive search are displayed in Table 1. It appears that the classical separation seems to be larger than the three other ones (absolute separation, separation of the real or imaginary parts), whose orders of growth seem similar.

For the same degrees and larger height, a fully exhaustive search becomes too time-consuming. Instead, we performed extensive experiments. The resulting record values are reported in Table 2, For degree 3 (resp. degree 4, degree 5), we computed up to height 200 (resp. height 120, height 30). As expected, polynomials with small separation tend to have larger height. To balance this bias, according to the form of the bounds on the separation, we filter the polynomials of interest by their quality, defined as $-\ln (S) / \ln (H)$ for a polynomial of height $H$ and separation $S$, and display only polynomials of "high quality". 


\begin{tabular}{|c|c|c|}
\hline$P(X)$ & abssep & $-\frac{\log \text { abssep }}{\log \mathrm{H}(P)}$ \\
\hline $2 X^{3}+X^{2}-X-1$ & $5.309 \mathrm{e}-2$ & 4.24 \\
\hline $13 X^{3}+11 X^{2}+8 X+5$ & $3.462 \mathrm{e}-5$ & 4.00 \\
\hline $8 X^{3}+7 X^{2}-9 X-17$ & $2.038 \mathrm{e}-5$ & 3.81 \\
\hline $17 X^{3}+9 X^{2}-7 X-8$ & $1.233 \mathrm{e}-5$ & 3.99 \\
\hline $17 X^{3}+9 X^{2}+7 X+8$ & $1.957 \mathrm{e}-5$ & 3.83 \\
\hline $102 X^{3}+97 X^{2}+71 X+40$ & $1.532 \mathrm{e}-8$ & 3.89 \\
\hline $153 X^{3}+97 X^{2}-71 X-60$ & $4.492 \mathrm{e}-9$ & 3.82 \\
\hline $71 X^{3}+112 X^{2}+153 X+181$ & $1.681 \mathrm{e}-9$ & 3.89 \\
\hline $181 X^{3}+153 X^{2}+112 X+71$ & $9.007 \mathrm{e}-10$ & 4.01 \\
\hline$X^{4}-X^{2}-2 X-3$ & $8.615 \mathrm{e}-4$ & 6.42 \\
\hline$X^{4}-X^{2}+2 X-3$ & $8.615 \mathrm{e}-4$ & 6.42 \\
\hline $3 X^{4}+3 X^{3}+X^{2}-2 X-4$ & $4.585 \mathrm{e}-5$ & 7.21 \\
\hline $4 X^{4}+2 X^{3}-X^{2}-3 X-3$ & $3.655 \mathrm{e}-5$ & 7.37 \\
\hline $5 X^{4}+3 X^{3}+X^{2}-X-3$ & $5.893 \mathrm{e}-5$ & 6.05 \\
\hline$X^{4}+6 X^{3}-7 X^{2}-5 X+6$ & $2.276 \mathrm{e}-6$ & 6.68 \\
\hline $6 X^{4}+5 X^{3}-7 X^{2}-6 X+1$ & $2.497 \mathrm{e}-6$ & 6.63 \\
\hline $11 X^{4}+7 X^{3}-X^{2}-10 X-16$ & $2.671 \mathrm{e}-8$ & 6.29 \\
\hline $16 X^{4}+10 X^{3}+X^{2}-7 X-11$ & $2.266 \mathrm{e}-8$ & 6.35 \\
\hline $3 X^{4}+6 X^{3}-4 X^{2}+3 X-18$ & $1.799 \mathrm{e}-8$ & 6.17 \\
\hline $18 X^{4}+3 X^{3}+4 X^{2}+6 X-3$ & $1.095 \mathrm{e}-8$ & 6.34 \\
\hline$X^{4}+40 X^{3}+11 X^{2}-14 X-55$ & $3.384 \mathrm{e}-11$ & 6.02 \\
\hline $55 X^{4}+14 X^{3}-11 X^{2}-40 X-1$ & $2.724 \mathrm{e}-11$ & 6.07 \\
\hline$X^{5}+X^{4}+2 X^{3}+3 X^{2}-2 X+2$ & $2.697 \mathrm{e}-5$ & 9.58 \\
\hline $2 X^{5}+X^{4}-X^{3}+X^{2}-X-1$ & $1.051 \mathrm{e}-3$ & 9.89 \\
\hline $2 X^{5}+X^{4}+X^{3}-2 X^{2}+2 X-2$ & $2.790 \mathrm{e}-3$ & 8.49 \\
\hline $2 X^{5}+X^{4}+X^{3}-X^{2}-X-1$ & $3.800 \mathrm{e}-3$ & 8.04 \\
\hline $2 X^{5}+X^{4}+2 X^{3}-2 X^{2}+X-2$ & $2.130 \mathrm{e}-3$ & 8.88 \\
\hline $2 X^{5}+X^{4}+2 X^{3}-X^{2}-X-2$ & $3.350 \mathrm{e}-3$ & 8.22 \\
\hline $2 X^{5}+X^{4}+2 X^{3}+2 X^{2}-2 X-2$ & $2.130 \mathrm{e}-3$ & 8.88 \\
\hline $2 X^{5}+2 X^{4}+X^{3}-X^{2}-2$ & $6.473 \mathrm{e}-4$ & 10.59 \\
\hline $4 X^{5}+2 X^{4}-4 X^{3}+3 X-2$ & $1.463 \mathrm{e}-6$ & 8.03 \\
\hline $8 X^{5}+5 X^{4}-4 X^{3}+4 X^{2}-5 X-4$ & $5.185 \mathrm{e}-8$ & 8.07 \\
\hline
\end{tabular}

Table 2: Polynomials with small absolute separation 


\begin{tabular}{rrlc}
\hline$n$ & height & abssep & $-\frac{\log \text { abssep }}{\log \mathrm{H}(P)}$ \\
\hline 2 & 12 & $5.093 \mathrm{e}-3$ & 2.12 \\
5 & 123 & $2.447 \mathrm{e}-6$ & 2.68 \\
10 & 2,340 & $4.643 \mathrm{e}-11$ & 3.36 \\
20 & $1,694,157$ & $1.690 \mathrm{e}-23$ & 3.66 \\
50 & $642,934,702,584,732$ & $8.146 \mathrm{e}-58$ & 3.86 \\
\hline
\end{tabular}

Table 3: Absolute separation for cubic polynomials $P_{n}$ from Proposition 3

Observing that cubic polynomials of high quality in Table 3, namely $13 x^{3}+$ $11 x^{2}+8 x+5$ and $181 x^{3}+153 x^{2}+112 x+71$, have roots in similar locations pushed us to refine our search in this vicinity and eventually led us to an unexpected family leading to a proof of optimality in the next section.

For polynomials of degree 4 up to height 120 , the results in Table 2 seem to suggest a bound of at most $O\left(\mathrm{H}(P)^{-6}\right)$, which should be reached by a realcomplex gap, as the complex-complex gap only gives $O\left(\mathrm{H}(P)^{-3}\right)$. This is far from the theoretical bound $O\left(\mathrm{H}(P)^{-12}\right)$ from Theorem 11. The best exponent we obtain by perturbations is -5 , see 3.4 .

For polynomials of degree 5 , we only have results up to height 30 and even those have been obtained by focusing in some cases of real-complex gap. Here, the bound on the height seems to be too small to observe a family. We have two polynomials with good quality that are relatively similar, namely $2 x^{5}+x^{4}-$ $x^{3}+x^{2}-x-1$ and $8 x^{5}+5 x^{4}-4 x^{3}+4 x^{2}-5 x-4$. No other polynomial close to these two are observed with height at most 30 .

\subsection{The case of degree 3}

Proposition 3. The family of cubic polynomials

$$
P_{n}(X)=p_{n}\left(3 X^{3}-2 X^{2}+4 X-6\right)+6 q_{n}\left(X^{3}-X^{2}+1\right) \in \mathbb{Z}[X],
$$

where $\left(p_{n} / q_{n}\right)_{n}$ is the sequence of convergents of the continued fraction expansion of $\sqrt{3}$, has the property that

$$
\text { abs sep }\left(P_{n}\right) \ll \mathrm{H}\left(P_{n}\right)^{-4}, \quad n \rightarrow \infty .
$$

Theorem 1 shows that abs $\operatorname{sep}\left(P_{n}\right) \gg \mathrm{H}\left(P_{n}\right)^{-4}$, so that the exponent -4 is optimal in degree 3 . The absolute separations of a few polynomials in that family are displayed in Table 3. The height of the polynomials $P_{n}$ increases exponentially with $n$.

Proof. It is readily checked that the bivariate polynomial

$$
P(X, Y)=\left(-\frac{1}{3} X^{2}+\frac{1}{2} X^{3}+\frac{2}{3} X-1\right) Y+X^{3}-X^{2}+1
$$


is such that for $Y=\sqrt{3}$, its 3 roots have absolute value exactly $\sqrt{3}-1$. A small perturbation $Y=\sqrt{3}+\epsilon$ sends the real root to

$$
\sqrt{3}-1+(2-\sqrt{3}) \epsilon+O\left(\epsilon^{2}\right)
$$

while the complex roots have absolute value

$$
\sqrt{3}-1+(2-\sqrt{3}) \epsilon+O\left(\epsilon^{2}\right)
$$

with a different constant in the $O()$ term so that the difference of the absolute values is $O\left(\epsilon^{2}\right)$. Evaluating $Y$ at a convergent $p / q$ of the continued fraction expansion of $\sqrt{3}$ leads to $\epsilon=|p / q-\sqrt{3}|<1 / q^{2}$, so that the difference of the absolute values of roots is $O\left(q^{-4}\right)$. The polynomial with integer coefficients obtained by normalizing $P(X, p / q)$ has coefficients growing asymptotically like $q$, giving -4 as a bound in the exponent.

Further experiments In an unsuccessful attempt to generalize the nice family of cubic polynomials in Proposition 3 to higher degrees, we analyzed more precisely the properties of the bivariate polynomial of Equation (2). A polynomial $a_{3} X^{3}+\cdots+a_{0}$ with simple roots, all of the same nonzero absolute value, has coefficients that satisfy

$$
a_{1}^{3} a_{3}-a_{0} a_{2}^{3}=0 .
$$

The set of such polynomials is therefore contained in an algebraic set of dimension 3 in $\mathbb{R}^{4}$. The question is to find a good point on this set whose perturbations behave well with respect to the absolute separation.

Polynomials in that set factor as $\left(a_{2} X+a_{1}\right)$ times a quadratic polynomial, whose discriminant has to be negative for its roots to have identical absolute value. This gives necessary and sufficient conditions: $a_{1} a_{2}<0 \leq a_{1} a_{3} \leq$ $a_{2}^{2} \leq 3 a_{1} a_{3}$. These conditions define a region on our algebraic set where all polynomials have 3 roots of identical absolute value.

A very special property possessed by the polynomial of Equation (2) is that in its case, the polynomial from Equation (3) factors as a square $\left(1-Y^{2} / 3\right)^{2}$. Adding this condition and forcing the perturbation of $Y$ to cancel the constant and linear coefficients of the expansion of the absolute separation finally leads to a 3-dimensional set of polynomials of which that of (2) is an instance:

$$
a\left(X^{3}+10 c^{3}\right)+b\left(3 X^{2}+6 c X\right)-\left(\left(X^{2}+4 c X\right) b+6 a c^{3}\right) \sqrt{3} .
$$

Polynomials in that family all have three roots of identical absolute value, and replacing $\sqrt{3}$ by convergents to its continued fraction expansion lead to an exponent 4 for their asymptotic absolute separation.

\subsection{Degrees 4 and 6}

For $d \in\{4,6\}$, we consider the polynomial $P=M\left(X^{d}-1\right)-Q_{d}(X)$, with $M$ an integer and $Q_{d}$ a polynomial of degree $d-1$ to be made precise later. As $M$ tends 
to infinity, the roots of $P$ tend to those of $X^{d}-1$. In particular, one of them tends to -1 and another one tends to $c_{d}$ a root of the $d$-th cyclotomic polynomial. The asymptotic expansion in powers of $1 / M$ of the difference between the absolute values of these roots can be obtained as follows. First the equation $P=0$ is rewritten as

$$
\frac{X^{d}-1}{Q_{d}(X)}=\frac{1}{M}
$$

In the neighbourhood of a root $\omega$ of $X^{d}-1$, the left-hand side behaves like

$$
\frac{d}{\omega Q_{d}(\omega)}(X-\omega)+\left(\frac{d(d-1)}{2 \omega^{2} Q_{d}(\omega)}-\frac{d Q_{d}^{\prime}(\omega)}{\omega Q_{d}(\omega)^{2}}\right)(X-\omega)^{2}+\cdots
$$

Power series inversion then gives the asymptotic behaviour of the corresponding root of Equation (4):

$$
X_{\omega}=\omega+\omega Q_{d}(\omega) \frac{1}{d M}+\left(\omega^{2} Q_{d}(\omega) Q_{d}^{\prime}(\omega)-\frac{(d-1)}{2} \omega Q_{d}(\omega)^{2}\right) \frac{1}{d^{2} M^{2}}+\cdots
$$

Substituting $\omega$ by $1 / \omega$ gives the expansion of the conjugate root and multiplying them gives the expansion of $\left|X_{\omega}\right|^{2}$. Finally, subtracting the values of this expansion for $\omega=-1$ and $\omega=c_{d}$ gives an expansion of the distance between the squares of these absolute values with coefficients that are polynomials in $c_{d}$ and in the coefficients of $Q_{d}$. Cancelling those coefficients up to order $1 / M^{d}$ gives a system of $d$ equations in the $d$ coefficients of $Q$. Up to multiplying $M$ by a constant, when $d \in\{4,6\}$, there is only one case when this system has integer solutions that do not correspond to $Q_{d}$ having a common factor with $X^{d}-1$, leading to the following.

Proposition 4. Let $d \in\{4,6\}$ and let $M$ be a positive integer. Consider the polynomials $P_{d, M}$ of degree $d$ defined by

$P_{d, M}=M\left(X^{d}-1\right)-Q_{d}(X)$, with $\left\{\begin{array}{l}Q_{4}(X)=X^{3}-X^{2}+X-5, \\ Q_{6}(X)=9 X^{5}-9 X^{4}-26 X^{3}-9 X^{2}+9 X-28,\end{array}\right.$

As $M$ tends to infinity, these polynomials have height $M$ and two roots $\alpha, \beta$ satisfying

$$
0<|| \alpha|-| \beta|| \ll \mathrm{H}\left(P_{d, M}\right)^{-d-1} .
$$

Absolute separations for a few polynomials in these families are given in Table 4

Proof. Once these polynomials have been found, the proof can be carried out by hand (and more easily with the help of a computer algebra system). The polynomial $P_{4, M}$ has roots

$$
\begin{aligned}
& z_{1}=1-\frac{1}{M}-\frac{2}{M^{2}}-\frac{11}{2 M^{3}}-\frac{71}{4 M^{4}}+O\left(\frac{1}{M^{5}}\right) \\
& z_{i}=i-\frac{i}{M}-\frac{1+4 i}{2 M^{2}}-\frac{4+11 i}{2 M^{3}}-\frac{66+143 i}{8 M^{4}}+O\left(\frac{1}{M^{5}}\right) .
\end{aligned}
$$




\begin{tabular}{rrlc}
\hline$d$ & height & abssep & $-\frac{\log \text { abssep }}{\log \mathrm{H}(P)}$ \\
\hline 4 & 10 & $3.716 \mathrm{e}-5$ & 4.43 \\
4 & 20 & $4.183 \mathrm{e}-7$ & 4.90 \\
4 & 50 & $2.653 \mathrm{e}-9$ & 5.05 \\
4 & 100 & $7.175 \mathrm{e}-11$ & 5.07 \\
4 & 500 & $2.055 \mathrm{e}-14$ & 5.07 \\
4 & 1000 & $6.335 \mathrm{e}-16$ & 5.07 \\
\hline 6 & $10^{2}$ & $3.336 \mathrm{e}-7$ & 3.24 \\
6 & $10^{3}$ & $1.373 \mathrm{e}-14$ & 4.62 \\
6 & $10^{4}$ & $1.267 \mathrm{e}-21$ & 5.22 \\
6 & $10^{5}$ & $1.257 \mathrm{e}-28$ & 5.58 \\
6 & $10^{10}$ & $1.256 \mathrm{e}-63$ & 6.29 \\
6 & $10^{20}$ & $1.256 \mathrm{e}-133$ & 6.65 \\
6 & $10^{30}$ & $1.256 \mathrm{e}-203$ & 6.76 \\
\hline
\end{tabular}

Table 4: Absolute separation for polynomials $P_{d, M}$ from Proposition 4

Taking the absolute values and subtracting shows that

$$
\left|z_{1}\right|-\left|z_{2}\right| \sim \frac{\left|z_{1}\right|^{2}-\left|z_{i}\right|^{2}}{2}=O\left(\frac{1}{M^{5}}\right) .
$$

The same reasoning applies to the polynomial $P_{6, M}$, with roots

$$
\begin{aligned}
z_{1} & =1-\frac{19}{3 M}-\frac{1235}{18 M^{2}}-\frac{240445}{162 M^{3}}+\cdots \\
z_{\frac{-1+i \sqrt{3}}{2}} & =\frac{-1+i \sqrt{3}}{2}-\frac{4(1-i \sqrt{3})}{3 M}-\frac{238-166 i \sqrt{3}}{9 M^{2}}-\frac{33383-13511 i \sqrt{3}}{81 M^{3}}+\cdots
\end{aligned}
$$

leading to

$$
\left|z_{1}\right|-\left|z_{\frac{-1+i \sqrt{3}}{2}}\right| \sim \frac{\left|z_{1}\right|^{2}-\left|z_{\frac{-1+i \sqrt{3}}{2}}\right|^{2}}{2}=O\left(\frac{1}{M^{7}}\right) .
$$

Perturbations of the other roots, namely $(1 \pm i \sqrt{3}) / 2$, lead to another system of equations for the coefficients of $Q_{6}$, but this system does not have integer solutions.

Further experiments Looking for similar polynomials using different products of cyclotomic polynomials also yields

$$
M\left(X^{2}-1\right)\left(X^{2}+X+1\right)-X^{3}+3 X+4
$$

with absolute separation in $\mathrm{H}^{-5}$. 
For $d=8$, the same computation only produces an exponent $-d+4$ : the polynomial system obtained when trying to cancel one more coefficient does not have any integer solution.

For odd degree $d$, the use of cyclotomic polynomials in this method only seems to reach exponent $d$ instead of $d+1$, with examples like

$M\left(X^{2}+1\right)(X-1)+X^{2}-2 X+3, \quad M\left(X^{2}-X+1\right)(X-1)+2 X^{2}-4 X-3$,

in degree 3 and

$$
\begin{aligned}
& M(X-1)\left(X^{2}+X+1\right)\left(X^{2}-X+1\right)+3 X^{4}-2 X^{3}+5 X^{2}-4 X+7, \\
& M(X-1)\left(X^{2}+1\right)\left(X^{2}-X+1\right)+2 X^{4}-8 X^{3}+13 X^{2}-12 X+7
\end{aligned}
$$

in degree 5.

\subsection{Degree 5}

For polynomials of degree 5 , exhaustive search cannot reach heights sufficient to exhibit the beginning of an asymptotic behaviour.

The perturbative approach remains possible, although the polynomial systems become rapidly too large for the Gröbner basis engine we use, which is Faugère's FGb [6]. We consider the polynomial $P=M R_{5}(X)-Q_{5}(X)$, with

$$
\begin{aligned}
& R_{5}(X)=\left(X^{2}+a X+r^{2}\right)\left(X^{2}+b X+r^{2}\right), \\
& Q_{5}(X)=X^{5}+q_{4} X^{4}+q_{3} X^{3}+q_{2} X^{2}+q_{1} X+q_{0},
\end{aligned}
$$

where all coefficients are unknowns, and expected to take rational values, with $a$ and $b$ in the interval $(-2 r, 2 r)$ so that $R_{5}$ has four roots of absolute value $|r|$. As in the previous case, the equation $P=0$ rewrites

$$
\frac{R_{5}(X)}{Q_{5}(X)}=\frac{1}{M}
$$

Expanding the left-hand side in the neighbourhood of solutions of the factors of $R_{5}$ and inverting the power series expansion gives expansions of the corresponding perturbed roots $X_{1}(\epsilon), X_{2}(\epsilon)$ of $P$. From there, the coefficients $c_{1, k}, c_{2, k}$ of $\left|X_{1}(\epsilon)\right|^{2}$ and $\left|X_{2}(\epsilon)\right|^{2}$ for $k$ from 1 to 5 are obtained. These coefficients are polynomials in $r, a, b$ and $q_{i}$ for $0 \leq i \leq 4$. The polynomial system $\left\{c_{1, k}=c_{2, k}, 1 \leq k \leq 5\right\}$ is unfortunately too big for a direct computation by Gröbner bases. Instead, we run a loop over possible integer values of $r, a, b$ in a given search range, and use Gröbner bases to determine whether each specialized system has (not necessarily integral) solutions and to determine the solution. If the solution happens to be rational, this gives a family with separation $O\left(\mathrm{H}(P)^{-6}\right)$. This is how the following family was found.

Proposition 5. Let $M$ be a positive integer and $P_{5, M}$ be defined by

$$
P_{5, M}=M R_{5}(X)-Q_{5}(X), \text { with }\left\{\begin{array}{l}
R_{5}(X)=\left(X^{2}-9 X+36\right)\left(X^{2}-11 X+36\right), \\
Q_{5}(X)=X^{5}-213 X^{3}+2404 X^{2}-11088 X+20736 .
\end{array}\right.
$$




\begin{tabular}{rlc}
\hline height & abssep & $-\frac{\log \text { abssep }}{\log \mathrm{H}(P)}$ \\
\hline $10^{10}$ & $7.165 \mathrm{e}-38$ & 3.81 \\
$10^{20}$ & $7.164 \mathrm{e}-98$ & 4.91 \\
$10^{50}$ & $7.164 \mathrm{e}-278$ & 5.56 \\
$10^{100}$ & $7.164 \mathrm{e}-578$ & 5.78 \\
$10^{200}$ & $7.164 \mathrm{e}-1178$ & 5.89 \\
$10^{500}$ & $7.164 \mathrm{e}-2978$ & 5.96 \\
\hline
\end{tabular}

Table 5: Absolute separation for polynomials $P_{5, M}$ from Proposition 5

As $M$ tends to infinity, this polynomial has height $O(M)$ and two roots $\alpha, \beta$ satisfying

$$
0<|| \alpha|-| \beta|| \ll \mathrm{H}\left(P_{5, M}\right)^{-6} .
$$

Again, given the polynomials, the proof is a direct computation of the asymptotic absolute separation. Absolute separations for a few polynomials in this family are given in Table 5 .

Note that actually, we get two one-parameter families of such polynomials with the same $R_{5}$ as above and

$$
\begin{gathered}
Q_{5}(X)=X^{5}+q X^{4}-(20 q+229) X^{3}+(2700+171 q) X^{2} \\
-(13104+720 q) X+(25920+1296 q), \\
Q_{5}(X)=X^{5}+q X^{4}-(20 q+213) X^{3}+(2404+171 q) X^{2} \\
-(11088+720 q) X+(20736+1296 q) .
\end{gathered}
$$

The family in the proposition is the special case $q=0$ of the second one.

\section{Conclusion}

From our experiments in low degree, it would be tempting to conjecture that a tight bound on the exponent of the absolute separation in degree $d$ is $-d-1$, but we have not been able to prove this, even for degrees 7 or 8 . Even the degree of the exponent of our lower bounds in Theorem 1 seems too high. The bounds in Theorem 1 are derived by considering arbitrary triples or quadruples of roots, without taking into account that some of them are conjugate, so this approach could make them pessimistic. More deeply, the cubic exponent seems to be inevitable for a purely algebraic approach: if $P=a_{0}+\cdots+a_{d} z^{d}$ is written $a_{d} \prod\left(z-\left(x_{k}+i y_{k}\right)\right)$, then $x_{k}, y_{k}$ and $x_{k}^{2}+y_{k}^{2}$ are generically algebraic of degree $d(d-1) / 2$ over $\mathbb{Q}\left(a_{0}, \ldots, a_{d}\right)$, with minimal polynomials having degree $d-1$ in the $a_{i}$ 's, whence again a cubic exponent. Obtaining better bounds thus probably needs more analytic tools.

Another question which is of a more analytic nature and more directly relevant for the complexity of algorithms on linear recurrences would be to determine a bound on the minimal distance between the two largest distinct absolute 
values of the roots. For any polynomial, this quantity is at least as large as the absolute separation, but does it have a different asymptotic behaviour?

On a related matter, Koiran recently used analytic arguments (Rolle's theorem and Baker's theory of linear forms in the logarithms of algebraic numbers) to give a bound on the (classical) root separation for trinomials, with a very small dependency on the degree [ 8 . It is not clear to us whether similar results also hold for the absolute separation.

\section{Ackowledgements}

W. Fang and B. Salvy were supported in part by FastRelax ANR-14-CE250018-01. A. Dujella and T. Pejković were supported by the Croatian Science Foundation under the project no. IP-2018-01-1313 and the QuantiXLie Center of Excellence, a project co-financed by the Croatian Government and European Union through the European Regional Development Fund - the Competitiveness and Cohesion Operational Programme (Grant KK.01.1.1.01.0004). Y. Bugeaud, A. Dujella, T. Pejković, and B. Salvy were supported in part by the FrenchCroatian bilateral COGITO project 'Approximation diophantienne et courbes elliptiques'.

\section{References}

[1] Bugeaud, Y., And Dujella, A. Root separation for reducible integer polynomials. Acta Arith. 162, 4 (2014), 393-403.

[2] Bugeaud, Y., Dujella, A., Pejković, T., and Salvy, B. Absolute real root separation. The American Mathematical Monthly 124, 10 (dec 2017), 930-936.

[3] Dubickas, A. On the distance between two algebraic numbers. Bulletin of the Malaysian Mathematical Sciences Society (2019), 1-16.

[4] Dubickas, A., And Sha, M. Counting and testing dominant polynomials. Exp. Math. 24, 3 (2015), 312-325.

[5] Evertse, J.-H. Distances between the conjugates of an algebraic number. Publ. Math. Debrecen 65, 3-4 (2004), 323-340.

[6] Faugère, J.-C. FGb: A Library for Computing Gröbner Bases. In Mathematical Software - ICMS 2010 (Berlin, Heidelberg, September 2010), K. Fukuda, J. Hoeven, M. Joswig, and N. Takayama, Eds., vol. 6327 of Lecture Notes in Computer Science, Springer Berlin / Heidelberg, pp. 84-87.

[7] Gourdon, X., And Salvy, B. Effective asymptotics of linear recurrences with rational coefficients. Discrete Mathematics 153, 1-3 (1996), 145-163. 
[8] Kolran, P. Root separation for trinomials. J. Symbolic Comput. 95 (2019), 151-161.

[9] Macdonald, I. G. Symmetric functions and Hall polynomials, 2nd ed. Oxford Mathematical Monographs. Oxford University Press, 1995.

[10] Mahler, K. An inequality for the discriminant of a polynomial. The Michigan Mathematical Journal 11 (1964), 257-262.

[11] Melczer, S., And Salvy, B. Symbolic-numeric tools for analytic combinatorics in several variables. In ISSAC'16: Proceedings of the 2016 ACM International Symposium on Symbolic and Algebraic Computation (New York, NY, USA, 2016), ACM, pp. 333-340.

[12] Melczer, S., And Salvy, B. Effective coefficient asymptotics of multivariate rational functions via semi-numerical algorithms for polynomial systems. Tech. Rep. 1905.04187, arXiv, 2019.

[13] Mignotte, M. Mathematics for Computer Algebra. Springer New York, 1992.

[14] Schönhage, A. Polynomial root separation examples. Journal of Symbolic Computation 41, 10 (2006), 1080-1090.

[15] Sha, M. Effective results on the Skolem problem for linear recurrence sequences. Journal of Number Theory 197 (2019), 228 - 249.

[16] Yap, C. K. Fundamental Problems in Algorithmic Algebra. Oxford University Press, New York, 2000. 